\title{
INTERMITTENT BEHAVIOR INDUCED BY ASYNCHRONOUS INTERACTIONS IN A CONTINUOUS DOUBLE AUCTION MODEL
}

\author{
KAZUTO SASAI $^{*,+, \|}$, YUKIO-PEGIO GUNJI ${ }^{\dagger, \S}$ and TETSUO KINOSHITA*, \\ ${ }^{*}$ Research Institute of Electrical Communication, \\ Tohoku University, \\ 2-1-1 Katahira, Aoba-ku, Sendai, Miyagi 980-8577, Japan \\ ${ }^{\dagger}$ Faculty of Science and Engineering, \\ Waseda University, \\ 3-4-1 Okubo, Shinjuku-ku, Tokyo 169-8555, Japan \\ kazuto@riec.tohoku.ac.jp \\ \$pegioyukio@gmail.com \\ \$kino@riec.tohoku.ac.jp
}

Received 24 May 2016

Revised 21 June 2017

Accepted 23 June 2017

Published 11 July 2017

\begin{abstract}
Continuous asynchronous trading activity is a key to understanding real-world market behavior. However, it is not easy to implement an agent-based computational market model because of the ambiguity between time and space. In this study, we use a model of asynchrony in a continuous double auction market in the form of noise and order restrictions to link inside- and outside- uncertainties in the economic system. Our model shows intermittent behavior with a small parameter value, which leads to the misapplication of the price-update rule, and consequently drives burst behavior. The statistical property of time development shows a similar tendency to that in previous empirical studies. Thus, it demonstrates the relationship between the asynchronous property and the complexity of economic systems.
\end{abstract}

Keywords: Continuous double auction; asynchronous trading; intermittency; multi-agent system; economic uncertainty.

\section{Introduction}

This study links intermittent behavior of economic systems and that of biological systems from the perspective of asynchronous interaction between agents. The

$\$$ Corresponding author.

|| Present address: 2-8-10, Itsutsubashi, Aoba-ku, Sendai, Miyagi, Japan.

This is an Open Access article published by World Scientific Publishing Company. It is distributed under the terms of the Creative Commons Attribution 4.0 (CC-BY) License. Further distribution of this work is permitted, provided the original work is properly cited. 
intermittency is a fundamental property of complex systems and is a system activity characterized by abrupt changes between the periods of quiescent low-level fluctuations and bursting high-level fluctuations. In the statistical analysis, a special characteristic of the behavior is that the probability distribution and the frequency distribution show the power-law distribution [46]. In recent years, many studies found out intermittency at various levels of measurements in economic systems such as foreign exchange markets [21, 44] and stock markets [39]. There are many models to explain intermittency in economic systems from the dynamical system approaches $[12,31]$ and the power-law distribution in price dynamics [18] and firm sizes [1]. As an alternative approach to macroscopic dynamical systems, there are the studies of agent-based models, which define the complex economic system by using many interactive agents $[28,60]$. The main goal of these studies is to reproduce the macroscopic dynamics of real-world markets resulting from interactions between microscopic agents with behavioral rules. The types of the power-law distribution produced by the agent-based models are investigated [15, 23, 61]. Further, some studies have examined the power-law nature of temporal characteristics such as phase transition or intermittency on simulated results [30, 47, 52]. Recently, Biondo et al. $[7,8]$ showed that the information diffusion process with small-world network yields burst behavior and concluded self-organized criticality induces the intermittent behavior of economic systems. However, since the result critically depends on the parameter of network structure, there remains the question why the real economic systems select such critical parameters? To answer the question, we need another conceptual model which explains intermittent nature in economic systems with the origin of criticality.

As a conceptual basis of complex chaotic nature in the economy, evolutionary economics is proposed as a means of considering the nonequilibrium properties of economic systems in the natural state $[17,45,56]$. As a similar direction, another new economic perspective, complexity economics, has been proposed by the Santa $\mathrm{Fe}$ Institute (SFI) [3, 50]. These approaches define an economic system as a self-referential system, which follows to the definition of autopoiesis [41]. In particular, the theory of micro-macro loop [57] emphasizes the self-referential view of economic systems. Although the perspective of the self-referential economy is a simple consequence of the nonlinear chaotic dynamics observed on the actual economic systems, however, it cannot completely describe how to connect a system with the outside of the economy. Hansen [29] mentions two types of uncertainty: that existing inside and that existing outside economic models. Inside-uncertainty refers to the ambiguity of the agents' choices, while outside-uncertainty includes our observations regarding the effects of factors that the model cannot describe. According to the discussion of Hansen, the model describing that the intermittent nature is possibly connected to the self-organized criticality focuses on the inside-uncertainty but not considers the observation as the outside-uncertainty. This problem is discussed in different literature [24, 27]. 
As a solution to the problem of how to define the outside of a system, some studies introduced the notion of endo-perspective in different schemes, e.g. internal measurement [40], endophysics [51] and heterarchy [24, 25, 53]. Endo-perspective assumes an observer is standing inside the world, then the observations of the observer cannot be ignored and thus the system (wholeness of the world) and the external environment (outside) are defined with the observations. In [25, 53], we define an abstract heterarchy as a dynamic system, which involves self-referencing, and frame the problem using logic-based chaotic maps. The model shows pulse-like intermittent behavior when the two chaotic maps act in an asynchronous coupling. In the biological context, the concept of asynchronous interaction between multiagents was implemented to the swarm model [26]. Because the self-referencing and frame problems correspond to the inside- and outside- uncertainties of a market, considering this asynchronous relationship is a key to understanding the intermittent behavior of markets. In this study, we apply the notion of asynchrony between the inside and outside of a market and propose a model that shows intermittent behavior. This reveals the importance of asynchrony and continuous time in understanding the complex properties of markets.

The rest of this paper is organized as follows. In Sec. 2, we explain the conceptual positioning of asynchronous trading over continuous time. In Sec. 3, we present a basic model of a continuous double auction (CDA) and explain our order-restriction proposal. In Sec. 4, we discuss the relationship between our model and the statistical properties of market behavior. Section 5 concludes the paper.

\section{Asynchrony Between the Inside and the Outside of a Market}

Traders trade asynchronously over continuous time in real-world markets. Nevertheless, the conventional models of the agent-based artificial markets are developed in discrete time and synchronous trading [48]. Some studies suggested the importance of asynchrony in markets, e.g. trade timing, decision-making and so on. Boer et al. [10] discussed the property of asynchronous trading in financial markets. Benhammada et al. [6] proposed controlling the time interval between cause and effect in a decision-making process to implement an asynchronous trading property in an artificial market system. Scalas et al. [54] investigated the effect of waiting time in financial markets. However, these studies did not consider the connection between the asynchrony and the outside (external environment). Here, we introduce a model of asynchronous trading under continuous time in a CDA market. In Japanese, a CDA mechanism is called "zaraba," which means that the buyer and seller can submit orders whenever they want, and if the orders are satisfiable, the agents execute the transaction immediately. There are two principles of priority in a zaraba market, price and time. The price priority states that if there are two buying orders, $A$ and $B$, they will be placed in order and if $p_{A}>p_{B}, A$ will be successful, while if the orders are placed sequentially, i.e., $t_{A}<t_{B}, A$ will again be successful. 
When we define a CDA market as an agent-based computational model, there is the difficulty of describing temporal and spatial dynamics simultaneously. Suppose a person who is willing to sell an item has two buy orders. He/she submits a sell order to sell at the price offered by these buy orders. Then, a moment later, he/she realizes that another buy order might be posted with a better price than the two existing orders, and thus decides to wait. However, this might not occur, and he/she might be better-off accepting the best current order. When he/she is making a rational choice at any given moment, it is necessary to know what all of the traders think. However, this is not possible in the real world. This is the well-known bounded-rationality problem [58]. Kahneman [35] claimed that decision-making innately involves ambiguity and uncertainty, which reads to self-referential cycle such that an agent cannot know the wholeness of the market, and the choice of individual agent is ambiguous. Conversely, we can consider another factor supporting the decision-making in the above example. Imagine that the person is watching a TV show that reports that Donald Trump has won the presidential election, and he/she decides to defer the sale of his/her assets. This decision is not caused by factors inside the market, but rather by an external factor that can change the trend in market behavior. This example corresponds to a famous effect of private and public information [43]. Because factors from external world of the market are inherently indefinite, this is another difficulty in modeling asynchronous trading. Therefore, the new model of asynchrony is necessary to connect inside (self-referencing) factors to outside (external world) factors on CDA markets.

In this study, we propose a model of asynchronous trading using probabilistic selection of orders. We call this order restriction, which corresponds to a modification of the order-book mechanism. Figure 1 shows a conceptual diagram of synchronous and asynchronous trading in CDA markets. As shown in Fig. 1(a), a simple trading model involves all agents submitting their orders simultaneously, and the contract price being determined by the correction of these orders, a form of auction mechanism discussed in [4]. Conversely, the order-book model [Fig. 1(b)] is a well-known mechanism used to determine contracts [8]. In the order-book model, asynchronous trading is interpreted to a stochastic selection of an active agent, who can make a decision at that time. However, because the selection of an agent is made sequentially, this does not represent time continuity. Reference [11] considers not only the appearance of a new order but also the disappearance of the remaining orders, and obtains a result that is close to the power-law nature of price transition that is evident in real-world markets. Our proposed asynchronous trading scheme is depicted in Fig. 1(c). Based on the hypothesis that it is possible to represent the changing order book by using a probabilistically determined set of orders, and that the changing book amplifies the perturbation caused by the uncertainty of the external world, we use an order set created stochastically at a given point in time and simultaneously consider the uncertainty of the external world. Thus, there is a possibility that the simultaneous consideration of a changing order book and perturbation from beyond the market sometimes leads to the overlapped uncertainty, 

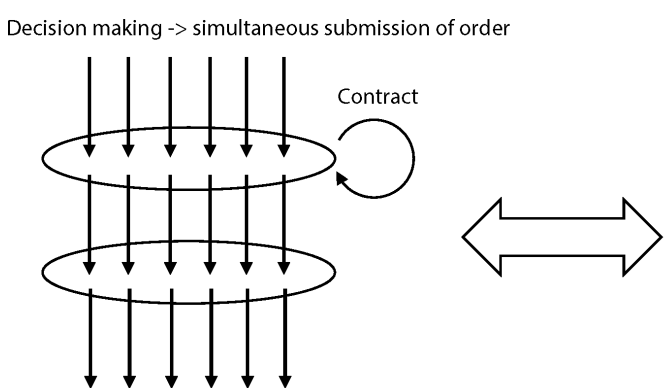

(a) Synchronous trading

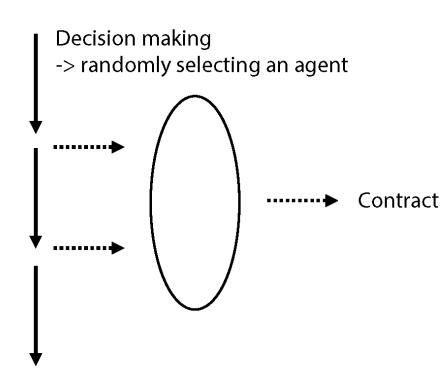

(b) Asyncronous trading (order book)
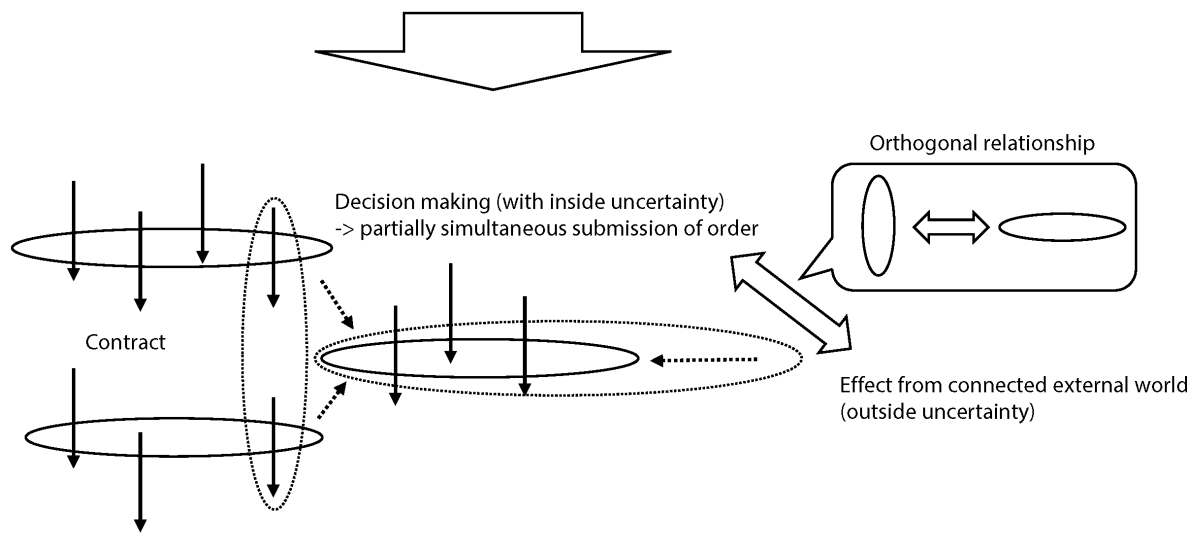

(c) Asynchronous trading (proposal)

Fig. 1. Comparisons of synchronous and asynchronous times: (a) and (b) are schematic diagrams of synchronous and asynchronous times in a CDA market model and (c) is a conceptual diagram of the asynchronous relationship between inside- and outside-uncertainties.

and which can link to Luhmann's double contingency [38,62] that is proposed to expand the theory of autopoiesis including the concept of otherness. We assume that the overlapped uncertainty causes the anomalous actions by agents such as overestimation, and consequently this can be a key to understanding real-world markets.

\section{CDA Model Based on Asynchrony}

The CDA model represents real-world markets such as stock exchanges [20]. The mechanism is simple: buyers and sellers simultaneously submit bid and ask orders, respectively. Several decision-making strategies for trading agents have been proposed, including the rational learning model [16] and the irrational random decision model [5, 22]. Although more complex models based on evolutionary learning mechanisms [2] and fuzzy logic [32] are available, these models focus on optimizing the rewards of an individual trader. Most double auction markets use asynchronous updates, although this is not related to the definition of asynchrony presented earlier. 
In this paper, we use a CDA model to demonstrate our proposed concept by reconstructing the rule of asynchronous trading.

\subsection{Basic model}

First, we explain our assumptions, which are based on an extension of a very simple model of a double auction proposed by [4]. The model we discuss here has two types of agents, i.e., buyers and sellers. There are equal numbers of each type, namely, $N$. In each time interval, a seller can only sell one item, but a buyer can bid for multiple items. Even though a double auction model should provide equal trading opportunities for all agents, here, we introduce a model in which multiple buyers bid to a seller to assert the boundary of the market and the effects from the external world. Thus, we start to build the model based on an extension of the single-seller auction model [4]. The basic model is defined as follows:

At the beginning of a trading term, a seller $j$ submits an asking price, $a^{t}(j)$, and a buyer $i$ decides on a bid price $b^{t}(i, j)$ for item $j$, i.e.,

$$
\begin{gathered}
b^{t}(i, j)=p_{b}^{t}(i)+v_{b}^{t}(i) \cdot \xi(j), \\
a^{t}(j)=p_{s}^{t}(j),
\end{gathered}
$$

where $p_{b}^{t}(i)$ is the basic price valuation of buyer $i, p_{s}^{t}(j)$ is the basic price valuation of seller $j$ and $v_{b}^{t}(i)$ is the fluctuation in competitive bidding behavior of the buyers. $\xi$ is a uniform random value. The basic price represents the estimated value of item $j$ by buyer $i$.

The seller of item $j$ decides on the contract price in term $t$ based on the maximum bid price. Buyer $i$ is a bidder for item $j$ if the asking price, $a^{t}(j)$, is less than the bid price, $b^{t}(i, j)$. Let $B^{t}(j)$ be a set of bidders for item $j$ such that

$$
B^{t}(j)=\left\{i \in N \mid b^{t}(i, j) \geq a^{t}(j)\right\} .
$$

Seller $j$ contracts with the bidder who bids the highest price as per the following equation:

$$
p^{t}(j)=\max _{i \in B^{t}(j)}\left\{b^{t}(i, j)\right\}
$$

There may be no bidder for an item because the asking price is higher than all of the bid prices. In this case, a deal is not finalized, and $B^{t}=\emptyset$. For a detailed explanation of the price determination process, we present the trading rule algorithm in Appendix A.

At the end of each term, the buyers and sellers update their prices in an attempt to obtain greater profits. Buyer $i$, who succeeded in buying at price $p_{b}^{t}(i)$, may want to offer a lower price next time. Thus, he/she determines an appropriate price of $d_{b}^{t}(i)=p_{b}^{t}(i)-v_{b}^{t}(i)$, where $v_{b}^{t}(i)$ is the speculative price fluctuation of buyer $i$. However, if he/she knows the trading results of the other agents, the speculative price $d$ should not be lower than the prices of buyers who failed to buy. Let $\mathcal{F}_{b}^{t}$ be the 
set of failed prices of buyers. Then, the moderation of the speculative price is represented by $\max \left(\mathcal{F}_{b}^{t}\right) \leq d_{b}^{t}(i)$. According to the moderation, the degree of the drop in price $v$ is reconsidered, and the price in $t+1$ is determined as follows:

$$
\begin{aligned}
& v_{b}^{t+1}(i)= \begin{cases}v_{b}^{t}(i) & \left(d_{b}^{t}(i) \geq \max \left(\mathcal{F}_{b}^{t}\right)\right), \\
\max \left(v_{\min }, \max \left(\mathcal{F}_{b}^{t}\right)-p_{b}^{t}(i)\right) & \left(d_{b}^{t}(i)<\max \left(\mathcal{F}_{b}^{t}\right)\right),\end{cases} \\
& p_{b}^{t+1}(i)=p_{b}^{t}(i)-v_{b}^{t+1}(i),
\end{aligned}
$$

where $v_{\min }$ is the bottom of fluctuation modulation. In contrast, buyer $i$, who failed to make a purchase in the previous period, may raise the price $p_{b}^{t}(i)$ to $d_{b}^{t}(i)=$ $p_{b}^{t}(i)+v_{b}^{t}(i)$ to increase the likelihood of success, while limiting the increase in price so that it is no greater than the price offered by buyers who were successful. Let $\mathcal{S}_{b}^{t}$ be the set of successful buyers' prices, where the limit can be described as $d_{b}^{t}(i) \leq \min \left(\mathcal{S}_{b}^{t}\right)$. Similarly, the reconsideration of price fluctuations $v_{b}$ is defined using Eqs. (5) and (6). A precise algorithmic definition of these price updates is presented in Appendix A.

Let us consider the seller $j$. If he/she succeeded in selling last time, he/she may want to raise the price, $d_{s}^{t}(j)=p_{s}^{t}(j)+v_{s}^{t}(j)$, where $v_{s}^{t}(j)$ is the speculative price fluctuation of seller $j$. However, because it is unreasonable to raise the price above the prices of sellers who failed to sell, the price reconsideration should be moderated to $d_{s}^{t}(j) \leq \min \left(\mathcal{F}_{s}^{t}\right)$. Then, the valuation in the next period is determined in the same manner by the buyers. As in the previous case, if seller $j$ fails to sell, the price may drop, i.e., $d_{s}^{t}(j)=p_{s}^{t}(j)-v_{s}^{t}(j)$, and he/she will consider the prices of the sellers who succeeded in selling, i.e. $\max \left(\mathcal{S}_{s}^{t}\right) \leq d_{s}^{t}(j)$, when determining the price for the next period (see Appendix A).

After the update of the prices, the next trading period commences. Table 1 shows the list of parameters used in the simulations. $v_{\min }$ is likely to be relatively small given the range of $v$. The basic model shows a monotonic increase in the price because the buyers bid prices that are slightly higher than the minimum price [Eq. (1)]. This reflects the asymmetry between the limited amount of items (stocks) and the unlimited desires (demands). However, the behavior does not correspond to overestimation or overconfidence, which are thought to be a cause of the bubbles and crashes [42], thus, we introduce the asynchrony of trading into this basic model to reveal the overlapped uncertainty. In the next subsection, we define the noise term, which is a

Table 1. List of parameters for the simulation experiment.

\begin{tabular}{ll}
\hline Parameter & Description \\
\hline$N=N_{b}=N_{s}=30$ & Numbers of both buyers and sellers \\
$p_{b}^{0} \in[0,5]$ & Initial valuation of buyers \\
$p_{s}^{0} \in[10,20]$ & Initial limit price of sellers \\
$v_{b}^{0} \in[1,11]$ & Initial value of price fluctuations among buyers \\
$v_{s}^{0} \in[1,11]$ & Initial value of price fluctuations among sellers \\
$v_{\min }=2$ & Minimum price fluctuation \\
\hline
\end{tabular}


metaphor for economic unrest such as deflation. The balance between competitive bidding and insecure risk hedging (price depression) induces a variety of equilibrium states.

\subsection{Trading with noise}

Conventional computational approaches to economics define external fluctuations as a noise term. For simplicity, we introduce a uniform price depression as a noise term. This is explained by the concept of bounded rationality [13, 36]. The equilibrium price state appears between strategic and noise traders [9, 14]. As with previous studies of noise traders, we introduce noise into the bidding price decision rule, which represents variations in the valuation of an item. The noise is represented using the probability of noise $\rho$ at each time point:

$$
p_{b, s}^{t+1}(i)=(1-\rho \cdot \xi) p_{b, s}^{t}(i) .
$$

The introduced noise parameter leads to an overall reduction in the market price and makes the formation of contracts easier. As mentioned in the previous subsection, the price increases monotonically in the basic model because of the buyers' competitive bidding. Figure 2(a) shows the behavior of the maximum contract price, $\max \left(p^{t}(j)\right)$, at each time point based on parameter $\rho$. At $\rho=0.0$, which corresponds to the basic model, the maximum contract price increases monotonically. If the noise parameter is small, the monotonic increase in price is gradually reduced, eventually reaching the equilibrium state $(\rho=0.002,0.02)$. Larger noise values such as $\rho=0.2$ lead to immediate convergence to an equilibrium around zero. Figure 2(b) illustrates the behavior when $\rho=0.2$. It can be seen that the price oscillates around the equilibrium point. Figure 2(c) shows the bifurcation map of $\rho$. Since the plot consists only of points in the range $1000<t<1200$, the figure shows the change in the equilibrium point. It can easily be seen that the equilibrium point falls as the level of noise increases. Even though this depends on the observed time interval, the equilibrium point approaches a value of 20 asymptotically [see Fig. 2(d)].

\subsection{Asynchronous trading represented by order restriction}

In this subsection, we implement a logical model of asynchronous trading, as mentioned in Sec. 2. The model we described in Sec. 3.2 is run in synchronous time because each auction is completed after collecting all of the bids. However, traders in real-world markets track changes in trends in real time and make instantaneous decisions. When a seller is willing to sell item $x$, he/she has three orders, $a, b$ and $c$, and he/she finally decides to form a contract at that time. In this case, a better offer could arrive later, meaning that he/she has lost money on the trade. Conversely, a buyer might have been willing to submit a better offer last time, but the seller rejected the order. In this case, the buyer has to raise the offer price next time. Considering these temporal aspects of traders' real-world behavior, we introduce the 


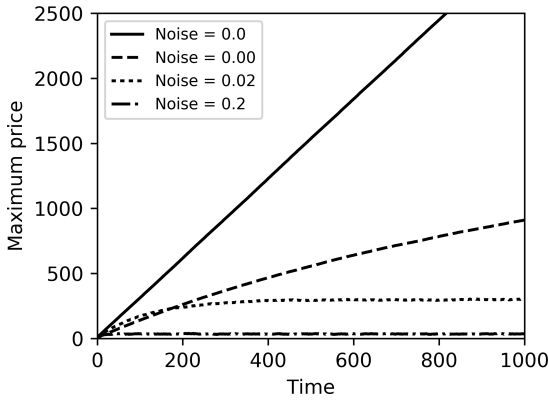

(a)

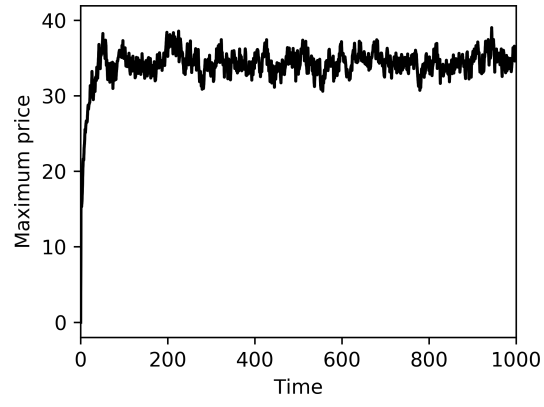

(b)

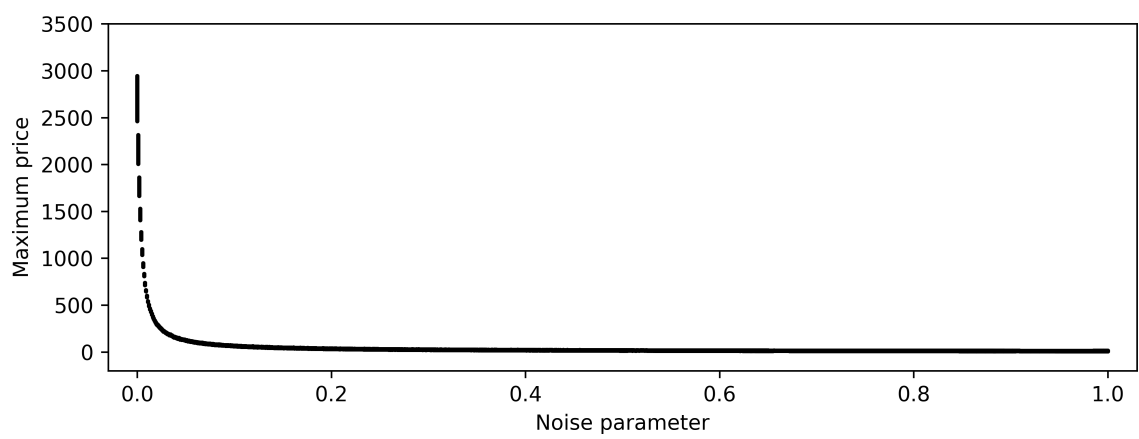

(c)

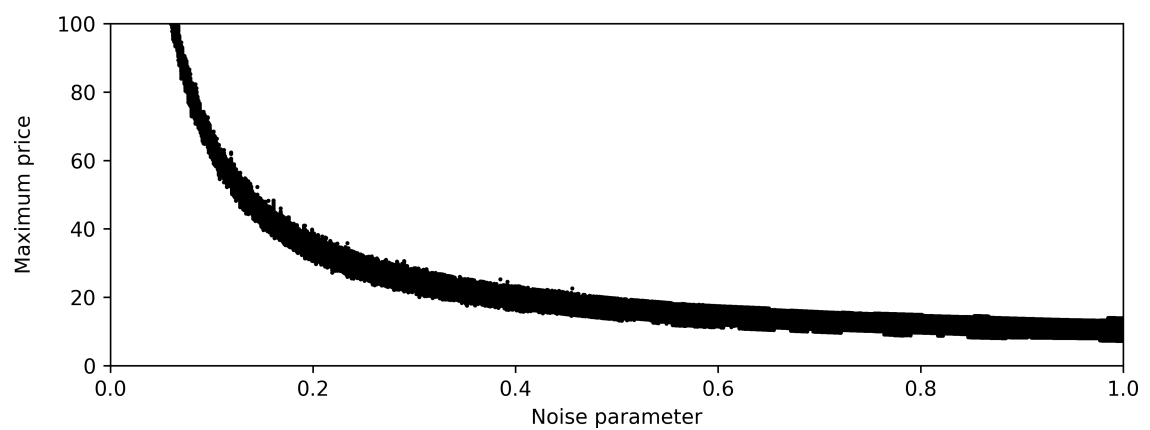

(d)

Fig. 2. (a) Time series based on $\rho$. (b) Detailed behavior when $\rho=0.2$. (c) Bifurcation map of the behavior of the maximum contract price against the noise parameter $\rho$ in the interval $1000<t<1200$ observed in 10 runs of 2000 step simulations with different initial conditions. (d) Detailed illustration of the monotonic increase in the change point toward equilibrium. The number of agents $N=30$.

order-restriction rule for late bidding, which means that a seller does not select a contract price from the complete list of bid prices, but from a list provided at an intermediate time point. Scalas et al. [54] studied the effect of waiting time on bidding and demonstrated the effects on the contract price of lengthening the 
decision-making time. However, in the present study, only the prices bid previously by buyers are considered. Implementing asynchrony can be considered to contrast with the experimental setup described by Scalas et al. [54]. The order-restriction rule allows for the incomplete correction of bids. Agents must decide upon their actions based on a partial set of bids, which results in the notion of asynchrony that we aim to demonstrate in this study. The order-restriction rule is defined as an additional parameter, $\sigma \in[0.0,1.0]$, which corresponds to the number of buyers who are probabilistically selected. In the conventional CDA model, an asynchronous update rule can be defined as the one that randomly selects an agent who bids or asks at a given moment [37]. If the selected agent can bid higher than the limit price set by the seller, the buyer and seller can enter into a contract. Because time flows synchronously in this model, it is not possible to represent the asynchronous selection of an activated agent.

Considering the order of arrival of bids to seller $j$, if an agent who can bid in asynchronous time is selected randomly, this can be represented by the randomly selected set of bidders with the stochastic parameter $\sigma$ such that, if $k$ bidders are selected, $\sigma=k / N$. Let $O^{t}(j)$ be the stochastically selected set of bids for seller $j$, i.e., $P\left(O^{t}(j)\right)=\sigma$, which indicates that the bids arrived before the deadline. The final contract price is defined as

$$
p^{t}(j)=\max _{i \in B^{t}(j) \cap O^{t}(j)}\left\{b^{t}(i, j)\right\} .
$$

Under this condition, the highest bid price may not be considered in the contract price decision if bidding is conducted relatively late.

Figure 3 shows the maximum contract price at each time point based on the order-restriction parameter $\sigma$ with noise $\rho=0.05$. The simulation setting is the same as in the previous subsection. Figures $3(\mathrm{a})$ and $3(\mathrm{~b})$ show the prices over time when $\sigma=0.01$ and $\sigma=0.03$, respectively. In this case, the intermittent price behavior is present in the first time period, which means that the effect of introducing the order restriction is critical to the characteristic behavior. As shown in Fig. 3(c), when $\sigma=0.05$, the behavior is similar to that in the equilibrium state with some noise [as shown in Fig. 2(c)], which means that the intermittent behavior only appears when $\sigma$ is small. In other words, few candidates are required to bid. Thus, we can expect that contracts are strictly inhibited when conditions are defined by a very high level of order restriction. Figure 3(d) shows the behavior at $\sigma=0.2$. This behavior is similar to that in the unrestricted order, $\sigma=1.0$ [as shown in Fig. 3(b)]. Thus, three types of characteristic behavior — intermittent, noisy and equilibrium - are observed based on the order-restriction parameter.

To investigate the effect of the changing behavior on the order-restriction parameter $\sigma$, we show a plot of the maximum contract price against $\sigma$ in Fig. 4 . Figure 4 (a) shows the behavior of the maximum contract price. The figure displays the compressed behavior of the individual parameter point. We only take points in the interval $1000<t<1200$ to display the equilibrium point when convergence occurs. 


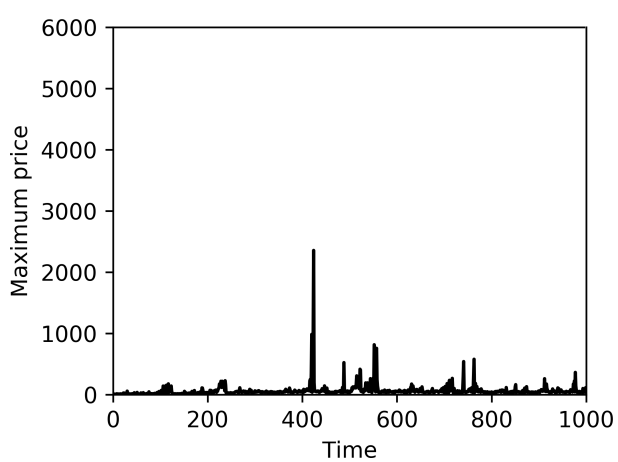

(a)

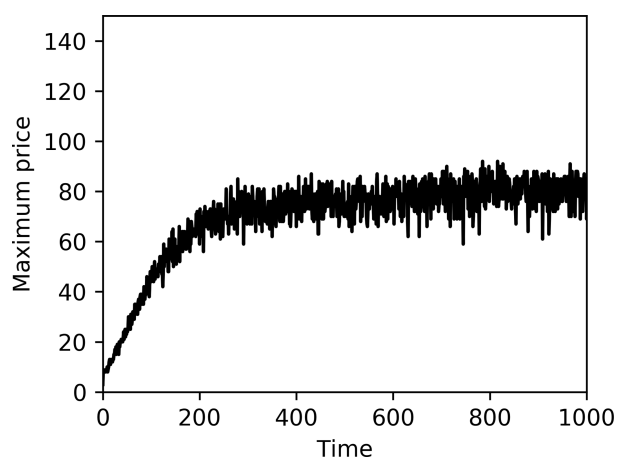

(c)

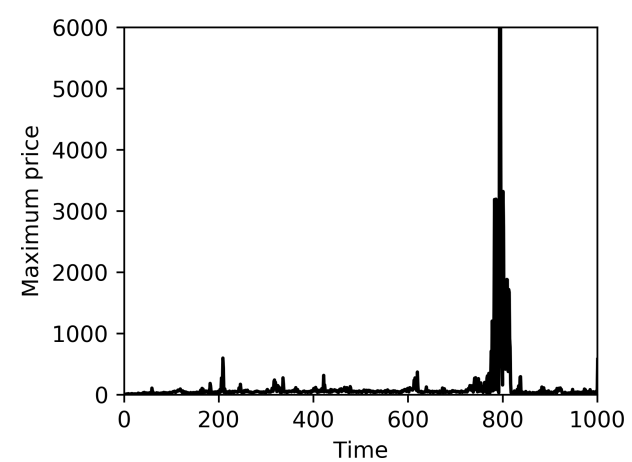

(b)

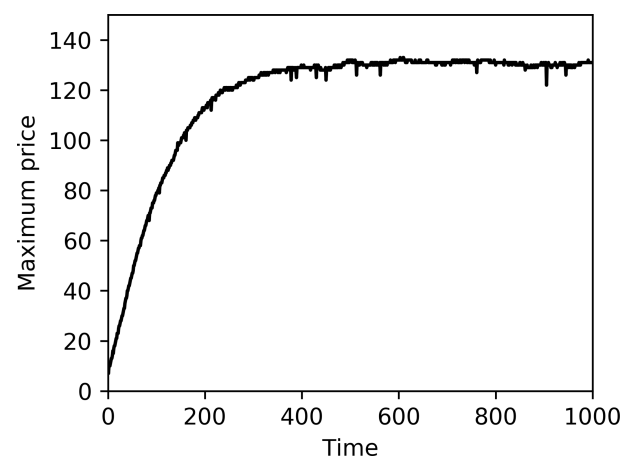

(d)

Fig. 3. Maximum contract prices at each time point with noise $\rho=0.02$. There are 30 agents $(N=30)$. When (a) $\sigma=0.01$ and (b) $\sigma=0.03$, intermittent behavior is observed. (c) When $\sigma=0.2$, order restriction creates noise around the equilibrium point. (d) When $\sigma=0.4$, the effect of the order restriction generally disappears. The behavior when there is a higher degree of noise is similar to the observations illustrated in (d).

In this figure, we limit the scale of the $y$-axis because the values at burst phase are too large. However, it should be remembered that there are burst points beyond the range shown in the figure. It is easy to find a critical point around $\sigma=0.1$. From Fig. 5, it can be seen that intermittent behavior occurs when $\sigma$ is less than this critical point. A greater value of $\sigma$ results in an equilibrium state, which grows asymptotically. Figure 4(b) shows the temporal difference in the maximum price $d^{t}=p_{\max }^{t}-p_{\max }^{t-1}$, which represents the magnitude of the price change. From the figure, it can be seen that the burst behavior gradually converges after the change point. Figure 4(c) shows the logarithmic expression of the highest price observed in 10 runs of 2000 step simulations, which provides more details in the area of intermittent behavior. The size of the burst is greater when $\sigma$ is almost zero. The change point is also found at around $\sigma=0.1$. Figure $4(\mathrm{~d})$ shows the frequency of bursts defined as $d^{t}>200$. The number of bursts increases according to the increase in $\sigma$, which falls around $\sigma=0.1$. 


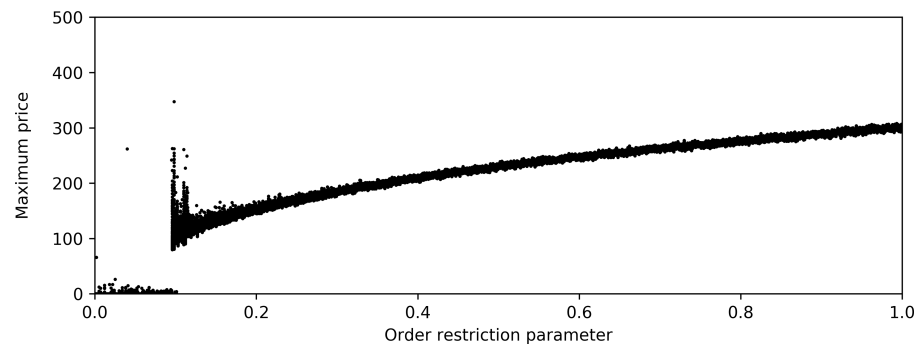

(a)

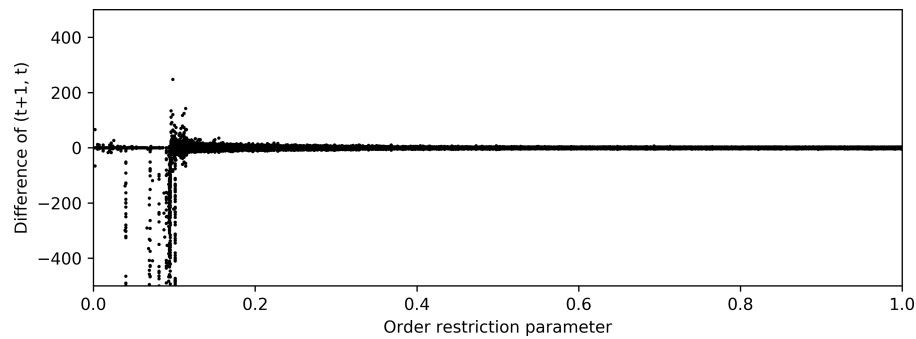

(b)

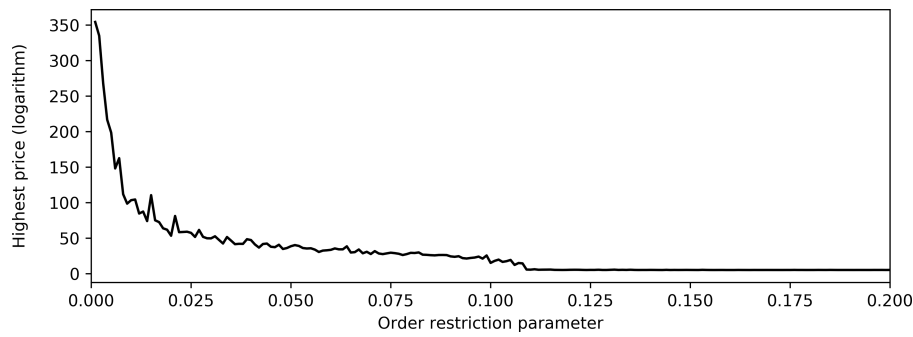

(c)

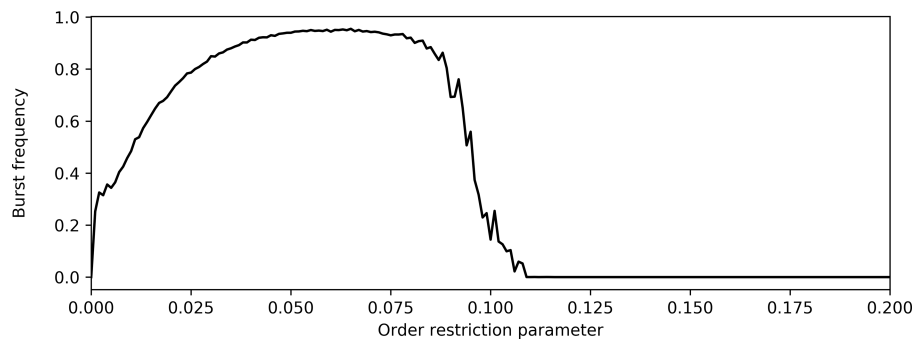

(d)

Fig. 4. Behavior of maximum contract prices $\max \left(P^{t}(j)\right)$ against the order-restriction parameter $\sigma$. (a) Bifurcation map of maximum contract prices. (b) Bifurcation map of the difference $d^{t}=$ $\max \left(p^{t}(j)\right)-\max \left(p^{t-1}(j)\right)$. (c) Highest price observed in 10 runs of 2000 step simulations with different initial conditions. (d) Normalized frequency of bursts, where a burst is counted if $d^{t}$ is higher than the threshold value of $\tau=200$. 


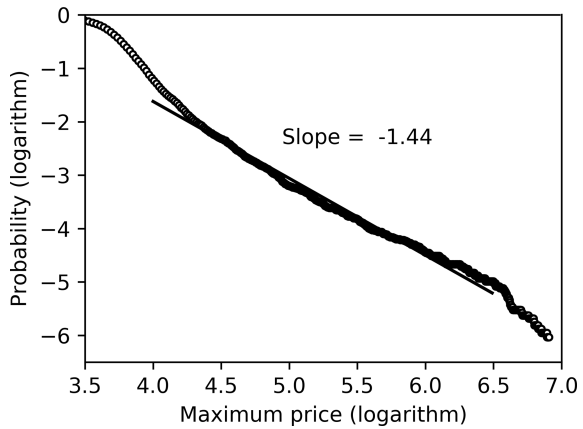

(a)

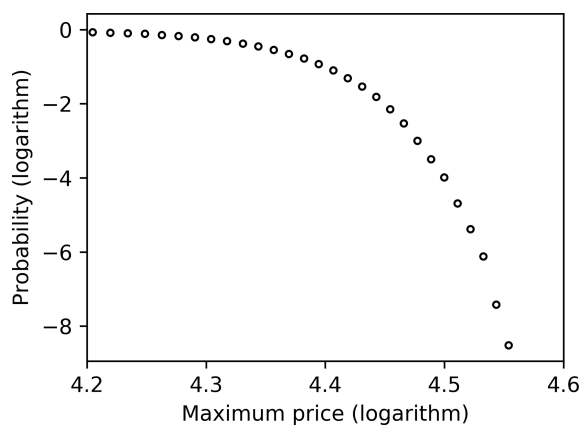

(c)

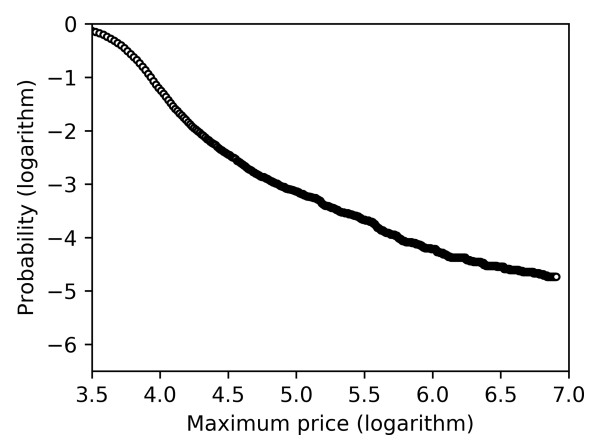

(b)

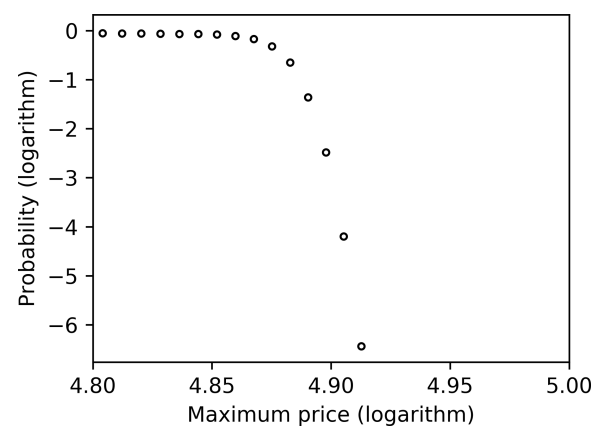

(d)

Fig. 5. Probability distribution of the average contract price at each time point with noise $\rho=0.05$ and the number of agents $N=30$. The horizontal axis indicates the maximum contract price variation and the vertical axis indicates the price probability calculated from 5000-step simulations. The axes are displayed using a logarithmic scale. (a) When the order-restriction parameter $\sigma=0.01$, a power-law distribution is shown with slope $\alpha \approx-1.44$. (b) When $\sigma=0.03$, a power-law-like distribution is shown. (c) When $\sigma=0.05$, a Poisson distribution is revealed. (d) When $\sigma=0.2$, the distribution is similar to that in (c) but closer to the random value.

From the results, it can be seen that there is a critical point, $\sigma \simeq 0.1$, between the intermittent state and the equilibrium state. Why does the burst occur at $\sigma<0.1$ ? The explanation is as follows. The most important reason for introducing order restriction is that a trader who arrives after the deadline is rejected. This leads to situations where either a buyer bids a high price but fails or a seller asks a low price but fails. Further, if $\sigma$ is sufficiently low, it is possible that no one can form a contract. This leads to a price increase for the buyer and a price decrease for the seller. If a buyer succeeds after spending a long time isolated from the market, the contract price is high. Because of Eqs. (5), (6), the high price is diffused throughout the market. This is why a small value of sigma leads to intermittent behavior. The noise that was added in Sec. 3.2 depresses the competitive increase in the price and brings about equilibrium. However, even though the small $\sigma$ increases the price, it does not achieve equilibrium with the noisy depression, but rather leads to intermittent bursts. 


\section{Statistical Properties of the Price Behavior}

As discussed above, our double auction model with order restriction produces significant changes in the temporal price trend. In this section, we investigate the statistical properties of the price transition. The price transition behavior of the most popular properties in real-world markets can be explained by a power-law distribution [59]. This distribution shows large fluctuations in prices as a result of significant trading volumes. However, this explanation does not account for the accumulated effect of small-scale trading activities. Our assertion in this study is that time asynchrony linked with incomplete wholeness can explain such market behavior.

Figure 5 shows the probability distribution of the average contract price $p_{\max }$ based on order restriction $\sigma$. The horizontal axis indicates the maximum contract price variation and the vertical axis indicates the price probability. Figure 5 (a) shows the case in which $\sigma=0.01$, which can be accurately explained using a power-law distribution with slope $\alpha=-1.44$. Figure $5(\mathrm{~b})$ shows the case in which $\sigma=0.03$, which almost reflects the power-law distribution, but does not do so precisely. Figures 5(c) and 5(d) show the cases in which $\sigma=0.05$ and $\sigma=0.2$, respectively. Both of these distributions fall to zero, which means randomness is dominant in these cases. This result implies that our model adequately captures the significant changes in the statistical properties of temporal behavior and the power-law distribution.

Furthermore, Gabaix et al. [19] showed that the statistical properties of stock volumes and returns in some real-world financial markets follow the power-law distribution, which is approximated using the following exponential function:

$$
P(x) \sim x^{-3 / 2},
$$

where $P(x)$ is the probability distribution of stock returns. The results of our model also indicate that the $\alpha=-3 / 2$ power-law distribution is similar to the results observed in a real-world market. Therefore, the simulated results are considered to be statistically comparable.

Furthermore, our model enables us to propose a way to improve the robustness of the system based on agents' cooperation. This proposal accords with the recognition of behavior with a power-law nature, called Lévy Flight, which implies greater exploratory capability and more resilience in the face of external perturbations.

This type of exploratory behavior is characterized by the power-law nature of the temporal property that was classified by Heagy and Platt [33]. These authors characterized intermittent behavior using the probability distribution of the time interval of the laminar phase, which corresponds to the intervals between bursts in our model. In this study, we define the burst intervals as follows:

$$
\Lambda(T)=\operatorname{Prob}\left(\bigcap_{j=1}^{T} p_{\max }^{j} \leq \tau \cap p_{\max }^{T+1}>\tau \mid p_{\max }^{1} \leq \tau\right),
$$




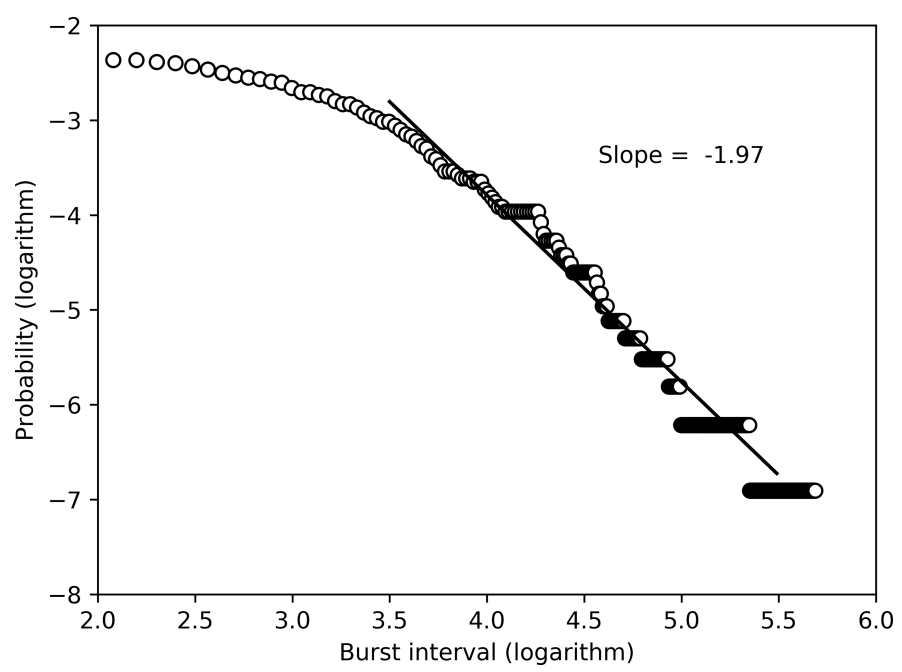

Fig. 6. Probability distribution of the intervals between the bursts of the average contract prices in a time sequence of 5000 step simulation. The horizontal axis indicates the duration of the intervals and the vertical axis indicates the probability of the intervals. $\rho=0.02$ and $\sigma=0.01$, with 30 agents $(N=30)$. The power-law distribution is shown with the slope $\beta=-1.97$.

where $\tau$ is the threshold that divides laminar and burst phases. In this study, we set $\tau=100$. Figure 6 shows the probability distribution of the burst intervals when $\sigma=0.01$ and $\rho=0.03$. In this case, the power-law distribution is presented with the slope $\beta=-1.97$. In our previous study, the power-law distribution was presented with the slope $\approx-2$ in the abstract model of the interplay among different levels [53], and it is known that Lévy Flight displays the same property [49]. In a recent empirical study, the intermittent behavior was found to follow a power-law distribution [52]. This result implies that few candidates are relevant to the important property of robustness.

\section{Discussion and Conclusion}

In this study, we propose a model describing the asynchronous property of trading in a CDA market. Introducing asynchrony, we describe the openness of an economic system, which explains the evolvability of the economy. Intuitively, economic trends are affected by both the economy itself and the world beyond. Even though economists know that both the macro- and micro-economic perspectives are important, we must choose one of these to construct an executable model of the economic system. For instance, in an agent-based model, we cannot design the behavior of the whole system, but only the rules that the agents must follow. In contrast, a macro-economic model cannot reflect each individual's activity. Therefore, we should start from the uncertainty of the two perspectives, i.e., the ambiguity of agent decisions and the 
external development of economics, and describe the interplay between them to explain the openness of an economic system.

We consider an agent-based model of a CDA market in which agents submit orders asynchronously. We start with inconsistency of time and prices, which implies that we must choose either the best current price or a future expectation. We implement the notions of noise-based decision-making and order restriction. The model shows convergence to an equilibrium state over a broad range of order restrictions. However, a high degree of restriction leads to intermittent behavior in the market. Under this condition, the situation exists whereby all of the orders fail, inducing misapplications of the price-update rule. The statistical properties of the price behavior are in line with real-world markets and the results of previous studies mentioned in Sec. 1. The intermittent behavior of a dynamic system appears within the critical area of the control parameter, which means that the intermittent behavior occurs between stable and unstable states. However, our model only has a single change point, which implies that the behavior is not critical, but appears universally under these conditions. Jen [34] also discusses the criticality of robustness compared with stability.

What is the implication of asynchronous time? This is intimately linked with our everyday life. Schwartz [55] explained in his book "The Paradox of Choice" using his personal experience. When he was young, the shops had only one type of jeans, but he was happy to buy them. However, nowadays there are various kinds of jeans, but he feels unhappy because the decision regarding which kind to buy is difficult. Here, from the perspective proposed in this study, the situation may occur in relation to the synchronous timing of decision-making, and it can be solved with asynchronous timing. Once you enter the shop, you may roam the floor, touch the jeans, talk with the salesperson and try them on. A choice is not a simple logical problem, but a changeable situation, and that is an implication of asynchronous time. Recently, this argument has become more serious in relation to choices on the Internet, because the online environment is continuously expanding, and it is becoming harder to make the right choices, according to Schwartz. We believe that a new understanding of asynchrony and uncertainty will help to guide our economic future. 


\section{Appendix A}

Algorithm A.1 shows the asynchronous trading rule, and Algorithm A.2 presents the price-update rule for the agents.

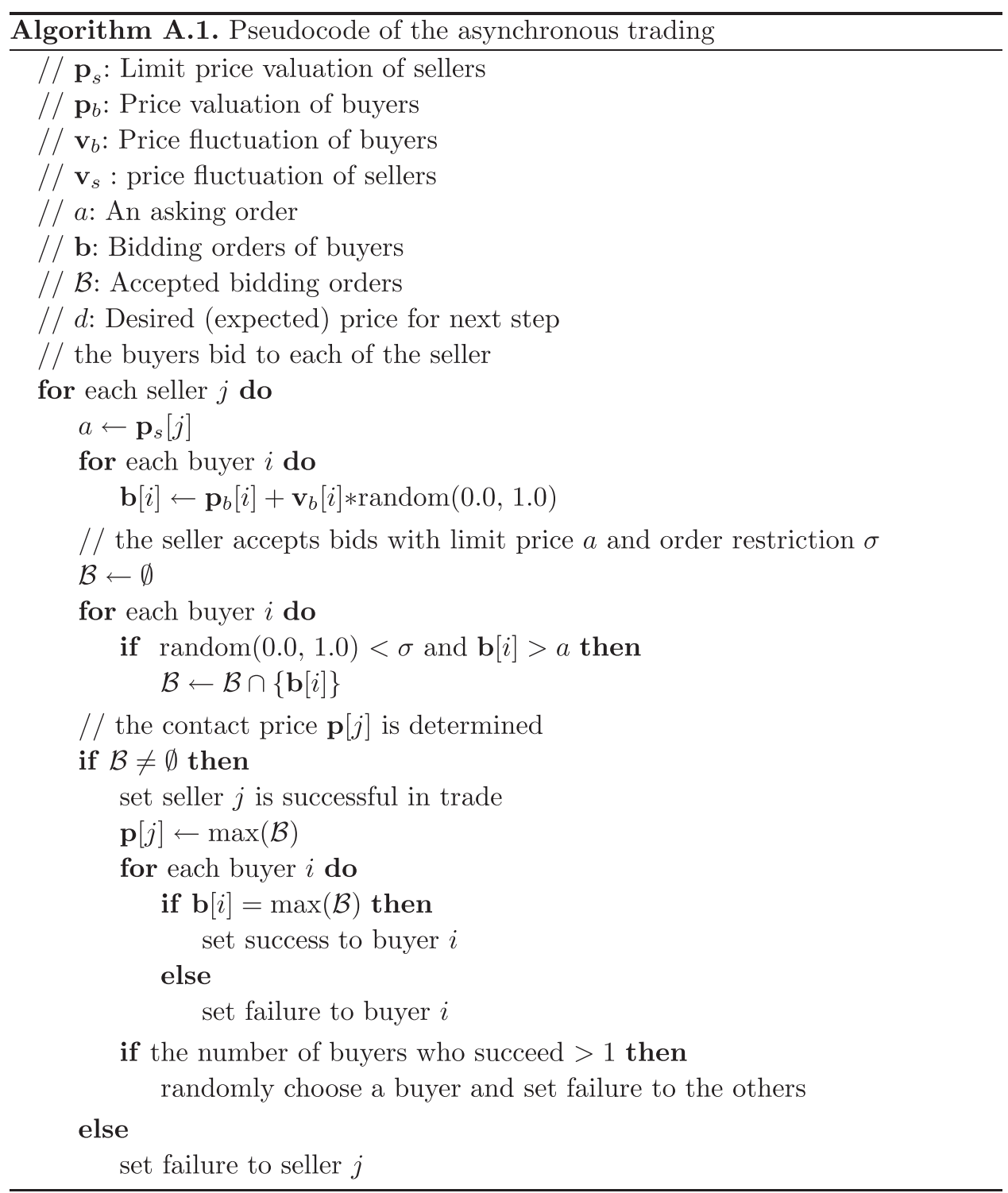




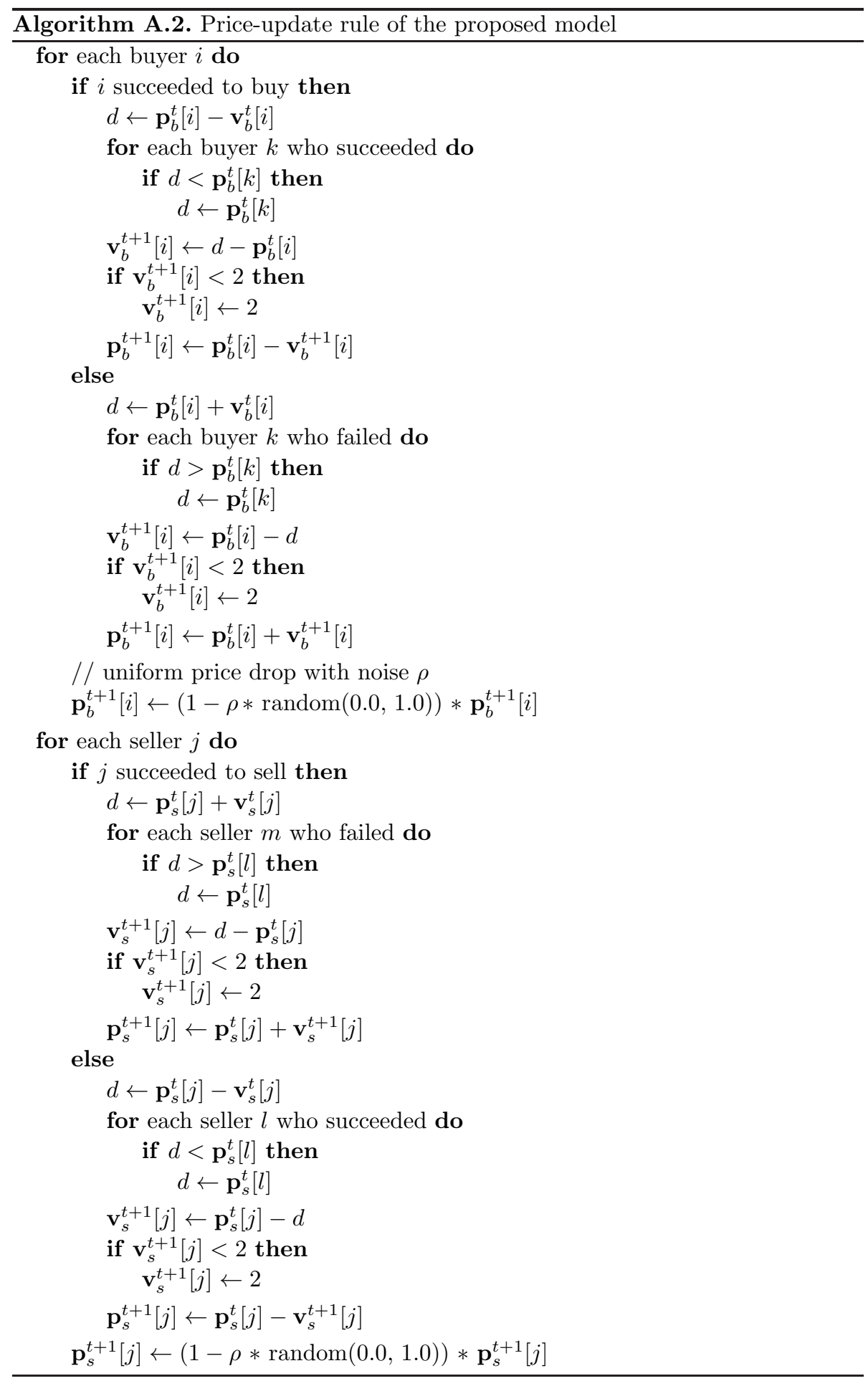




\section{References}

[1] Anderson, C., Long Tail: Why the Future of Business is Selling Less of More (Hyperion, New York, 2006).

[2] Anufriev, M., Arifovic, J., Ledyard, J. and Panchenko, V., Efficiency of continuous double auctions under individual evolutionary learning with full or limited information, J. Evol. Econ. 23 (2013) 539-573.

[3] Arthur, W. B., Complexity and the Economy (Oxford University Press, Oxford, 2015).

[4] Bagnall, A. and Toft, I., Autonomous adaptive agents for single seller sealed bid auctions, Auton. Agent. Multi-Agent Syst. 12 (2006) 259-292.

[5] Bak, P., Paczuski, M. and Shubik, M., Price variations in a stock market with many agents, Physica A 246 (1997) 430-453.

[6] Benhammada, S., Amblard, F. and Chikhi, S., An asynchronous double auction market to study the formation of financial bubbles and crashes, New Gener. Comput. 35 (2017) $129-156$.

[7] Biondo, A. E., Pluchino, A. and Rapisarda, A., Modeling financial markets by selforganized criticality, Phys. Rev. E 92 (2015) 042814.

[8] Biondo, A., Pluchino, A. and Rapisarda, A., Order, book, financial markets and selforganizing criticality, Chaos Solitons Fractals 88 (2016) 196-208.

[9] Blume, L. E., How noise matters, Games Econ. Behav. 44 (2003) 251-271.

[10] Boer, K., Kaymak, U. and Spiering, J., From discrete-time models to continuous-time, asynchronous modeling of financial markets, Comput. Intell. 23 (2007) 142-161.

[11] Challet, D. and Stinchcombe, R., Analyzing and modeling 1+1d markets, Physica A 300 (2001) 285-299.

[12] Chian, A. C. L., Complex Systems Approach to Economic Dynamics (Springer-Verlag, Berlin, 2007).

[13] Conlisk, J., Why bounded rationality? J. Econ. Lit. 34 (1996) 669-700.

[14] De Long, J. B., Shleifer, A., Summers, L. H. and Waldmann, R. J., Noise trader risk in financial markets, J. Polit. Econ. 98 (1990) 703-738.

[15] Feng, L., Li, B., Podobnik, B., Preis, T. and Stanley, H. E., Linking agent-based models and stochastic models of financial markets, Proc. Natl. Acad. Sci. USA 109 (2012) 8388-8393.

[16] Friedman, D., A simple testable model of double auction markets, J. Econ. Behav. Organ. 15 (1991) 47-70.

[17] Friedman, D., Evolutionary economics goes mainstream: A review of the theory of learning in games, J. Evol. Econ. 8 (1998) 423-432.

[18] Gabaix, X., Power laws in economics: An introduction, J. Econ. Perspect. 30 (2016) 185-206.

[19] Gabaix, X., Gopikrishnan, P., Plerou, V. and Stanley, H. E., A theory of power-law distributions in financial market fluctuations, Nature 423 (2003) 267-270.

[20] Garman, M. B., Market microstructure, J. Financ. Econ. 3 (1976) 257-275.

[21] Ghashghaie, S., Breymannn, W., Peinke, J., Talkner, P. and Dodge, Y., Turbulent cascades in foreign exchange markets, Nature 381 (1996) 767-770.

[22] Gode, D. K. and Sunder, S., What makes markets allocationally efficient? Q. J. Econ. 112 (1997) 603-630.

[23] Gontis, V. and Kononovicius, A., Consentaneous agent-based and stochastic model of the financial markets, PLoS ONE 9 (2014) e102201.

[24] Gunji, Y. P. and Kamiura, M., Observational heterarchy enhancing active coupling, Physica D 198 (2004) 74-105.

[25] Gunji, Y. P., Sasai, K. and Wakisaka, S., Abstract heterarchy: Time/state-scale re-entrant form, Biosystems 91 (2008) 13-33. 
[26] Gunji, Y. P., Nishiyama, Y. and Adamatzky, A., Robust soldier crab ball gate, Complex Syst. 20 (2011) 93-104.

[27] Gunji, Y. P., Self-organized criticality in asynchronously tuned elementary cellular automata, Complex Syst. 23 (2014) 55-69.

[28] Hamil, L. and Gilbert, N., Agent-Based Modeling in Economics (Wiley, 2015).

[29] Hansen, L. P., Uncertainty outside and inside economic models, J. Polit. Econ. 122 (2014) 945-987.

[30] Harmon, D., Lagi, M., de Aguiar, M. A. M., Chinellato, D. D., Braha, D., Epstein, I. R. and Bar-Yam, Y., Anticipating economic market crises using measures of collective panic, PLoS ONE 10 (2015) e0131871.

[31] Hommes, C. H., Economic dynamics, in Encyclopedia of Nonlinear Science, ed. Scott, A. (Routledge, New York, 2005), pp. 4-13.

[32] He, M., Leung, H. F. and Jennings, N. R., A fuzzy-logic based bidding strategy for autonomous agents in continuous double auctions, IEEE Trans. Knowl. Data Eng. 15 (2003) 1345-1363.

[33] Heagy, J. F. and Platt, N., Characterization of on-off intermittency, Phys. Rev. E 49 (1994) 1140-1150.

[34] Jen, E., Stable or robust? What's the difference? Complexity 8 (2013) 12-18.

[35] Kahneman, D., Maps of bounded rationality: Psychology for behavioral economics, Am. Econ. Rev. 93 (2003) 1449-1475.

[36] Knudsen, C., Equilibrium, perfect rationality and the problem of self-reference in economics, in Rationality, Institutions and Economic Methodology, eds. Mäki, U., Gustafsson, B. and Knudsen, C. (Routledge, 1993), pp. 133-170.

[37] Ladley, D., Zero intelligence in economics and finance, Knowl. Eng. Rev. 27 (2012) 273286.

[38] Luhmann, N., Social Systems (Stanford University Press, Stanford, 1995).

[39] Mantegna, R. N. and Stanley, H. E., Scaling behavior in the dynamics of an economic index, Nature 376 (1995) 46-49.

[40] Matsuno, K., Protobiology: Physical Basis for Biology (CRC Press, Boca Raton, 1998).

[41] Maturana, H. R. and Valera, F. J., Autopoiesis and Cognition: The Realization of the Living (D. Reidel Publishing Company, 1980).

[42] Michailova, J. and Schmidt, U., Overconfidence and bubbles in experimental asset markets, J. Behav. Financ. 17 (2016) 280-292.

[43] Morris, H. S. and Shin, S., Social value of public information, Am. Econ. Rev. 92 (2002) $1521-1534$.

[44] Müller, U. A., Dacorogna, M. M., Olsen, R. B., Pictet, O. V., Schwartz, M. and Morgenegg, C., Statistical study of foreign exchange rates, empirical evidence of a price change scaling law and intraday analysis, J. Bank. Financ. 14 (1990) 1189-1208.

[45] Nelson, R. R. and Winter, S. G., An Evolutionary Theory of Economic Change (Belknap Press, 1985).

[46] Ott, E., Chaos in Dynamical Systems (Cambridge University Press, Cambridge, 1993).

[47] Ouyang, F. Y., Zheng, B. and Jiang, X. F., Intrinsic multi-scale dynamic behaviors of complex financial systems, PLoS ONE 10 (2015) e0139420.

[48] Palmer, R. G., Arthur, W. B., Holland, J. H., LeBaron, B. and Tayler, P., Artificial economic life: A simple model of a stock market, Physica D 75 (1994) 264-274.

[49] Reynolds, A. M., On the anomalous diffusion characteristics of membrane-bound proteins, Phys. Lett. A 342 (2005) 439-442.

[50] Rosser, J. B., On the complexities of complex economic dynamics, J. Econ. Perspect. 13 (1999) 169-192. 
[51] Rössler, O. E., Endophysics, in Real Brains: Artificial Minds, eds. Casti, J. L. and Karlquist, A. (North-Holland, 1987), pp. 25-46.

[52] Rypdal, M., Sirnes, E., Løvsletten, O. and Rypdal, K., Assessing market uncertainty by means of time-varying intermittency parameter for asset price fluctuations, Physica A 392 (2013) 3335-3343.

[53] Sasai, K. and Gunji, Y. P., Heterarchy in biological systems: A logic-based dynamical model of abstract biological network derived from time-state-scale re-entrant form, Biosystems 92 (2008) 182-188.

[54] Scalas, E., Kaizoji, T., Kirchler, M., Huber, J. and Tedeschi, A., Waiting times between orders and trades in double-auction markets, Physica A 366 (2006) 463-471.

[55] Schwartz, B., The Paradox of Choice: Why More Is Less (Ecco, 2004).

[56] Shiozawa, Y., Evolutionary economics in the 21st century: A manifest, Evol. Inst. Econ. Rev. 1 (2004) 5-47.

[57] Shiozawa, Y., Nakajima, Y., Matsui, H., Koyama, Y., Taniguchi, K. and Hashimoto, F., Artificial Market Experiments with the U-Mart System (Springer Science \& Business Media, 2008).

[58] Simon, H., Models of Man, Social and Rational: Mathematical Essays on Rational Human Behavior in a Social Setting (Wiley, New York, 1957).

[59] Smith, E., Farmer, J. D., Gillemot, L. and Krishnamurthy, S., Statistical theory of the continuous double auction, Quant. Financ. 3 (2003) 481-514.

[60] Tesfatsion, L. and Judd, K. L. (eds.), Handbook of Computational Economics Vol. 2 (Elsevier, 2006).

[61] Tseng, J. J., Lin, C. H., Lin, C. T., Wang, S. C. and Li, S. P., Statistical properties of agent-based models in markets with continuous double auction mechanism, Physica $A$ 389 (2010) 1699-1707.

[62] Vanderstraeten, R., Persons, Luhmann and the theorem of double contingency, J. Class. Sociol. 2 (2002) 77-92. 